\title{
Accuracy of High-Resolution Computed Tomography Chest in Diagnosing Pulmonary Tuberculosis by taking AFB culture findings as Gold Standard
}

\author{
MUHAMMAD AMIN ${ }^{1}$, MUSSERAT JAVED ${ }^{2}$, AFSHAN NOREEN ${ }^{3}$, MARIA MEHBOOB ${ }^{4}$, NAZAHAT PASHA ${ }^{5}$, UMAIMA \\ MAJEED ${ }^{6}$ \\ ${ }^{1}$ Assistant Professor of Radiology, $(\mathrm{CH} \& \mathrm{ICH})$ Children Hospital and Institute of Child Health, Multan \\ ${ }^{2}$ Women Medical Officer, Radiology Department CH\&ICH Multan \\ ${ }^{3}$ Assistant Professor Radiology CH\&ICH Multan \\ ${ }^{4}$ Post Graduate Registrar Radiology $\mathrm{CH} \& \mathrm{ICH}$ Multan. \\ ${ }^{5}$ Senior Registrar Radiology $\mathrm{CH} \& \mathrm{ICH}$ Multan \\ ${ }^{6}$ Senior Registrar Radiology $\mathrm{CH} \&$ ICH Multan \\ Correspondence to: Dr. Muhammad Amin, Cell: 03336121755
}

\begin{abstract}
Aim: To determine the accuracy of high resolution CT Chest (HRCT) for diagnosis of pulmonary tuberculosis (PTB) by taking AFB culture findings as gold standard.

Methods: This validation study was conducted in patients referred for HRCT Chest for diagnosis of pulmonary tuberculosis in OPD/ Radiology unit of $\mathrm{CH} \& \mathrm{ICH}$ within duration of 6 months from September-2020 to February 2021. Patients with suspicion of PTB were included. In all patients, sputum samples were obtained and the AFB test was performed for diagnosis of PTB. After that high resolution CT Chest (HRCT) was performed for diagnosis of PTB.

Results: Mean age was $54.67 \pm 12.36$ years. Male population $54 \%$ and female was $46 \%$. AFB culture results for pulmonary. TB was positive in 44 patients and was negative in 56 patients. HRCT findings were positive for pulmonary TB in 46 patients and were negative in 54 patients (Table 1). The sensitivity of HRCT was $81.8 \%$, specificity of HRCT was $82.1 \%$, PPV was $78.3 \%$ and NPV was $85.2 \%$ (Table 2 ).

Conclusion: HRCT Chest has a sufficient accuracy for PTB diagnosis taking AFB culture results as gold standard. So HRCT Chest can be opted as imaging modality of choice in patients having mixed plain radiographs findings. This will aid in early identification and starting treatment of PTB.

Keywords: Accuracy, high resolution computed tomography, pulmonary tuberculosis.
\end{abstract}

\section{INTRODUCTION}

Pulmonary tuberculosis (TB) is a highly spread infectious disease of the developing world. It is of major concern for both developing and developed world and it is commonest cause of death due to infectious diseases, nearly causing 3 million deaths/year. ${ }^{1}$ According to WHO TB is the most neglected communicable diseases pandemic. ${ }^{2}$ TB is also highly prevalent in Pakistan. Patients with active infection present with fever, productive cough, with/without hemoptysis, weight loss and night sweats. ${ }^{3}$ According to reported up-to $95 \%$ death due to TB occur in poor countries of Asia, Africa and South America. ${ }^{4}$

The gold standard test for diagnosis of active TB is microbiological detection of acid fast bacillus (AFB), but this test has low sensitivity for sputum smear 46 to $74 \%$ and up-to $95 \%$ for sputum culture. According to National data the rate of detection of active TB is 10 to $22 \%$ among suspected cases $^{5,6}$.

Chest radiograph is the first most common radiological imaging performed in TB patients. on chest $\mathrm{X}$ rays pulmonary TB manifests as pleural effusion, consolidation, enlarged lymph nodes and military nodules. However, some of these features also present in post-TB patients. ${ }^{7,8}$ Recent studies have reported the utility of CT scan for evaluation and diagnosis of active TB especially in

Received on 13-01-2021
Accepted on 23-05-2021

patients with mixed radiology findings. ${ }^{9}, 10$ Therefore, this study is designed to determine the accuracy of high resolution CT (HRCT) Chest for diagnosis of pulmonary tuberculosis (PTB) taking AFB culture findings as gold standard.

\section{METHODS}

This validation study was conducted in patients referred for HRCT in OPD/Radiology unit of Children Hospital and Institute of Child Health Multan within duration of 6 months from Sep-2020 to Feb-2021. Patients with suspicion of pul. TB i.e. those presenting with productive cough, sputum, fever, weight loss and night sweats were included. While patients taking anti-tuberculosis treatment were excluded.

Data regarding routine investigations, sputum smear results, and plain radiographs were obtained for each patient.

In all patients, sputum samples were obtained and the AFB test was performed on Löwenstein-Jensen (LJ) medium, in case of mycobacterium growth on culture medium the test was labelled positive otherwise negative.

After that high resolution CT Chest (HRCT) was performed and following findings on HRCT were considered active pulmonary TB; presence of cetrilobular nodules, consolidation, branching modules (tree shaped), pleural effusion and lymph adenopathy. 
For data analysis we used SPSS v23 software. We formulated $2 \times 2$ table for determination of sensitivity, specificity, positive predictive value (PPV), andYnegative predictive value (NPV).

\section{RESULTS}

Mean age was $54.67 \pm 12.36$ years. Male population $54 \%$ and female was $46 \%$. AFB culture results for pul. TB was positive in 44 patients and was negative in 56 patients. HRCT Chest findings were positive for pulmonary TB in 46 patients and were negative in 54 patients (Table 1).

The sensitivity of HRCT was $81.8 \%$, specificity of HRCT was $82.1 \%$, PPV was $78.3 \%$ and NPV was $85.2 \%$ (Table 2).

Table1. Study Variables.

\begin{tabular}{|l|l|}
\hline Age & $54.67 \pm 12.36$ \\
\hline Gender & 54 \\
\hline Male & 46 \\
\hline Female & 56 \\
\hline AFB Culture Results & 44 \\
\hline Negative & \multicolumn{2}{|l|}{} \\
\hline Positive & 54 \\
\hline HRCT Results & 46 \\
\hline Negative & \\
\hline Positive &
\end{tabular}

Table 2. Accuracy of HRCT Chest Taking AFB Culture Findings As Gold Standard.

\begin{tabular}{|l|l|l|l|}
\hline \multirow{2}{*}{ TB on HRCT } & \multicolumn{2}{|l|}{ TB on AFB Culture } & \multirow{2}{*}{ Total } \\
\cline { 2 - 3 } & Yes & No & \\
\hline Yes & 36 & 10 & 46 \\
\hline No & 08 & 46 & 54 \\
\hline Total & 44 & 56 & 100 \\
\hline Sensitivity $=81.8 \%$ & \multicolumn{2}{|l}{} \\
\hline Specificity $=82.1 \%$ & Positive predictive value (PPV) $=78.3 \%$ \\
\hline Negative predictive value (NPV) $=85.2 \%$ \\
\hline
\end{tabular}

\section{DISCUSSION}

TB is a common communicable disease in Pakistan. TB can affect any organ, the commonest organ of involvement are lungs. Sputum smear is the first-line test for initial diagnosis of PTB but it's a slow test and can take up-to a week for final results. Similarly, AFB culture also takes several weeks to give final outcomes. ${ }^{11}$ While the imaging studies can produce results within minutes to hours and can evaluate treatment responses and can also detect complications associated with PTB. ${ }^{11}$

The initial reported of use of HRCT in detection of PTB have produced promising results and therefore can prove to be a good imaging modality for diagnosis and determining the response of PTB treatment, and a normal HRCT can help to exclude the possible cause of illness. ${ }^{12}$

In this study, we find that HRCT is $78.6 \%$ sensitive for determining PTB. While a previous study reported $96.4 \%$ sensitivity of HRCT and another study reported $82.7 \%$ sensitivity of HRCT. ${ }^{13}, 14$ A study from Pakistan by Rasheed et al. reported HRCT has a sensitivity of $89.09 \%$, specificity of $79.25 \%$, PPV of $81.67 \%$ and NPV of $87.50 \%$. ${ }^{15}$ This difference in accuracy of HRCT in different studies many be due to differences in epidemiology and risk factors that may affect accuracy. The other possible reason is the experience of radiologists and the parameters evaluated during CT imaging.

The potential limitation of present study is the limited sample size. The other limitation is that this study was conducted in a population having high prevalence of PTB. Still more studies are needed to be conducted containing larger populations and in mutli-centers to determine the accuracy of HRCT in a more reliable manner.

\section{CONCLUSION}

HRCT has a sufficient accuracy for PTB diagnosis taking AFB culture results as gold standard. So HRCT can be opted as imaging modality of choice in patients having mixed plain radiographs findings. This will aid in early identification and starting treatment of PTB.

\section{REFERENCES}

1. Glaziou P, Floyd K, Raviglione MC. Global Epidemiology of Tuberculosis. Semin Respir Crit Care Med. 2018;39(3):271-85.

2. Qadeer E, Fatima R, Yaqoob A, Tahseen S, UI Haq M, Ghafoor A, et al. Population Based National Tuberculosis Prevalence Survey among Adults (>15 Years) in Pakistan, 2010-2011. PLoS One. 2016;11(2):e0148293-e.

3. Wei Z, Zhang X, Wei C, Yao L, Li Y, Zhang X, et al. Diagnostic accuracy of in-house real-time PCR assay for Mycobacterium tuberculosis: a systematic review and meta-analysis. BMC Infect Dis. 2019;19(1):701.

4. Akhtar S, White F, Hasan R, Rozi S, Younus M, Ahmed F, et al Hyperendemic pulmonary tuberculosis in peri-urban areas of Karachi, Pakistan. BMC Public Health. 2007;7:70.

5. Khan M, Mashood A, Qureshi W, Ibrar K. Tuberculosis-disease pattern and the sputum microscopy yield. Pak J Chest Med. 2005;11:11-9.

6. Iqbal R, Shabbir I, Waseem T. Screening of AFB in sputum by concentration or direct smear method. Pak J Med Res. 2000;39:35-6.

7. Nachiappan AC, Rahbar K, Shi X, Guy ES, Mortani Barbosa EJ, Jr., Shroff GS, et al. Pulmonary Tuberculosis: Role of Radiology in Diagnosis and Management. Radiographics. 2017;37(1):52-72.

8. Burrill J, Williams CJ, Bain G, Conder G, Hine AL, Misra RR. Tuberculosis: a radiologic review. Radiographics. 2007;27(5):1255-73.

9. Nakanishi M, Demura Y, Ameshima S, Kosaka N, Chiba Y, Nishikawa $\mathrm{S}$, et al. Utility of high-resolution computed tomography for predicting risk of sputum smear-negative pulmonary tuberculosis. Eur $\mathrm{J}$ Radiol. 2010;73(3):545-50.

10. Kim J, Lee IJ, Kim JH. CT findings of pulmonary tuberculosis and tuberculous pleurisy in diabetes mellitus patients. Diagnostic and interventional radiology (Ankara, Turkey). 2017;23(2):112-7.

11. Bhalla AS, Goyal A, Guleria R, Gupta AK. Chest tuberculosis: Radiological review and imaging recommendations. Indian J Radiol Imaging. 2015;25(3):213-25.

12. Hantous-Zannad S, Zidi A, Néji H, Attia M, Baccouche I, Ben MiledM'rad K. [The role of imaging in thoracic tuberculosis]. Rev Pneumol Clin. 2015;71(2-3):93-109.

13. Yeh JJ, Yu JK, Teng WB, Chou CH, Hsieh SP, Lee TL, et al. Highresolution CT for identify patients with smear-positive, active pulmonary tuberculosis. Eur J Radiol. 2012;81(1):195-201.

14. Raghuvanshi V, Sood RG, Jhobta A, Sarkar M, Tomar A, Khanna S. Use of High-Resolution Computed Tomography (HRCT) in Diagnosis of Sputum Negative Pulmonary Tuberculosis. Turkish thoracic journal. 2016;17(2):59-64.

15. Rasheed W, Qureshi R, Jabeen N, Shah HA, Naseem Khan R Diagnostic Accuracy of High-Resolution Computed Tomography of Chest in Diagnosing Sputum Smear Positive and Sputum Smear Negative Pulmonary Tuberculosis. Cureus. 2020;12(6):e8467 